\title{
Increased physical activity is not enough to recover astrocytic population from dark-rearing. Synergy with multisensory enrichment is required
}

\author{
Harkaitz Bengoetxea ${ }^{1}{ }^{*}$, Naiara Ortuzar ${ }^{1}$, Irantzu Rico-Barrio ${ }^{1}$, José Vicente Lafuente ${ }^{1}$ and \\ Enrike G. Argandoña ${ }^{2}$
}

${ }^{1}$ Laboratory of Experimental Neuroscience, Department of Neuroscience, Faculty of Medicine and Odontology, University of the Basque Country, Leioa, Spain
${ }^{2}$ Unit of Anatomy, Department of Medicine, University of Fribourg, Fribourg, Switzerland

\section{Edited by:}

Lavinia Alberi, University of Fribourg,

Switzerland

\section{Reviewed by:}

Hari S. Sharma, Uppsala University, Sweden

Francesca Prestori, University of

Pavia, Italy

Abigail Kerr, Illinois Wesleyan

University, USA

\section{*Correspondence:}

Harkaitz Bengoetxea, Laboratory of Experimental Neuroscience,

Department of Neuroscience, Faculty of Medicine and Odontology,

University of the Basque Country, Barrio Sarriena, S/N, 48940 Leioa, Spain

e-mail: hbengoetxea@gmail.com
Elimination of sensory inputs (deprivation) modifies the properties of the sensory cortex and serves as a model for studying plasticity during postnatal development. Many studies on the effects of deprivation have been performed in the visual cortex using dark-rearing as a visual deprivation model. It induces changes in all cellular and molecular components, including astrocytes, which play an important role in the development, maintenance, and plasticity of the cortex, mediated by cytokines which have been termed angioglioneurins. When one sense is deprived, a compensatory mechanism called cross-modal plasticity increases performance in the remaining senses. Environmental enrichment is so far the best-known method to compensate sensorial deprivation. The aim of this work is to study the effects of exercise alone, and of an enriched environment combined with exercise, on astroglial population in order to observe the effects of exercise by itself, or the potential synergistic effect during the rat visual system development. Pregnant Sprague-Dawley rats were raised in one of the following rearing conditions: in total darkness and enriched environment conditions with physical exercise, and in total darkness with voluntary physical exercise. Astrocytic density was estimated by immunohistochemistry for S-100 $\beta$ protein and quantifications were performed in layer IV. The somatosensorial cortex barrel field was also studied as control. Our main result shows that an enriched environment combined with voluntary physical exercise manages to reverse the negative effects induced by darkness over the astroglial population of both the visual and the somatosensory cortices. On the other hand, exercise alone only produces effects upon the astroglial population of the somatosensory cortex, and less so when combined with an enriched environment.

Keywords: environmental enrichment, physical exercise, dark-rearing, astrocyte, cross-modal plasticity, neurogliovascular unit, angioglioneurins

\section{INTRODUCTION}

In most species sensory systems are immature at birth and their postnatal development is refined by sensory inputs which induce changes at behavioral, functional, cellular, and molecular levels (Greenough et al., 1987; Bengoetxea et al., 2012; Levelt and Hübener, 2012). Changes reach their maximum during the critical period (Hensch, 2004, 2005), a time window specific for each sensorial cortex. For the visual system of rats, this period starts at the end of the third postnatal week, peaks during the fourth week, and ebbs away from the end of the sixth week (Fagiolini et al., 1994). Although most studies have been focused on the neuronal component of the cortex, experience-mediated changes occur in neurons, glia, and vessels that constitute the so called neurogliovascular unit (Argandoña et al., 2012). These three cellular elements are closely related, constituting de facto the functional unit of the CNS.

Maturation of the sensory system is not only mediated by neuronal activity and cortical circuits (Hensch and Fagiolini, 2005), but by a plethora of elements including angioglioneurins
(Lafuente etal., 2012) - cytokines that have proliferative and protective effects on all components of the neurogliovascular unit. These molecules include factors initially described as neurotrophic factors, such as the brain derived neurotrophic factor (BDNF); angiogenic factors, such as the vascular endothelial growth factor (VEGF); or metabolic factors, such as the insulin-like growth factor (IGF) or erythropoietin (EPO; Zacchigna et al., 2008).

Modification of the properties in the sensory cortex by means of elimination of the sensory input (deprivation) is a widely used model for the study of plasticity during postnatal development (Fagiolini et al., 1994; Argandoña and Lafuente, 1996; Frasnelli et al., 2011). Most studies analyzing the effects of deprivation have been performed in the visual cortex using dark-rearing as a visual deprivation model. It has been described that dark-rearing causes changes in all neurogliovascular components, inducing a decrease in neuronal and astroglial population (Argandoña and Lafuente, 1996, 2000; Argandoña et al., 2003), among others.

Despite the fact that glial cells are by far the most numerous brain cell types, their role has long been regarded as of 
passive support. Since the seventies it is well known that astrocytes are mainly responsible for the development and function of the blood-brain barrier (Peterson et al., 2011). Recently the "tripartite synapse" paradigm (Perea etal., 2009; Ben Achour and Pascual, 2012) demonstrates that astroctyes are structurally and functionally related to neurons in a bidirectional way. Thus, astrocytes play a crucial role in the development, maintenance, plasticity, and function of the cortex, taking part in the control of the balance between energy demand and vascular support in the so-called neuro-glio-vascular coupling (Figley and Stroman, 2011). Considering the pivotal role of astrocytes in brain homeostasis and the strong metabolic cooperation existing between neurons and astrocytes, astrocytic dysfunction might contribute to neurodegenerative processes or vice versa, being both cellular types important unto each other (McGann et al., 2012).

A common feature for all sensory deprivation is the compensatory cross-modal plasticity which leads to an increased performance in the remaining senses when someone is subjected to sensory deprivation (Rauschecker, 1995; Merabet and Pascual-Leone, 2010). When sensory deprivation occurs, the corresponding cortex can be reactivated by the sensory system. The best-known method for compensating visual deprivation is environmental enrichment. Rearing in complete darkness from birth has major effects on the development of the visual cortex which can be reverted if animals are dark-reared in complex environments (Bengoetxea et al., 2008). Bartoletti et al. (2004) showed that environmental enrichment promotes the consolidation of the visual cortical connections and the development of visual acuity in dark-reared rats, and compensates for the effects of dark-rearing on a chondroitin sulfate proteoglycan-based perineuronal net establishment of the visual cortex. Visual deprivation also has effects at a molecular level. Our previous work showed that levels of the archetypal angioglioneurin VEGF diminish with visual deprivation, while an enriched environment helps to increase these levels up to control values (Bengoetxea et al., 2008).

Exercise has been considered essential for enriched environments to induce structural and functional changes (Will et al., 2004; Xie et al., 2013), but its role is still somewhat controversial, as exercise has been demonstrated to have effects by itself (Mustroph et al., 2012). In contrast, some behavioral studies have described a lack of differences between inclusion and non-inclusion of exercise (Pietropaolo et al., 2006). Most of these studies have been performed at the hippocampus and few results have been reported on sensory cortical areas.

The goal of this work was an in-depth analysis of the cross-modal reorganization of the visual cortex of rats, during development, in order to determine if the recovery of electrical properties and the closure of the critical period described by other authors can be correlated to quantitative effects on the astrocyte population. Therefore, we aimed to isolate exercise from the enriched environment and compare the importance of exercise alone or the potential synergistic effect of a combination of an enriched environment with exercise on the recovery of the astroglial population in the visual and the somatosensory cortices.

\section{MATERIALS AND METHODS ANIMALS AND HOUSING}

Pregnant Sprague-Dawley rats were raised in different rearing conditions (Figure 1):

(1) Dark rearing with exercise (DR-Ex): rats raised in total darkness with unlimited access to a running wheel (2-3 rats per cage).

(2) Dark rearing in an enriched environment with exercise (DR-EE-Ex): rats raised in total darkness in enriched environment conditions and unlimited access to a running wheel. The enriched environment consists of a large cage $(720 \mathrm{~mm} \times 550 \mathrm{~mm} \times 300 \mathrm{~mm})$ furnished with colorful toys and differently shaped objects (shelters, tunnels) that were changed every 2 days (8-10 rats per cage; data for this group were retrieved from our previous work "Argandoña et al. (2009)" in order to reduce the use of animals).

During postnatal development, the following age groups were studied: P21, P28, P35, P42, P49, P56, and P63. Rats were raised from birth in each of the described rearing conditions. Weaning was carried out at P21, when males and females were separated and both sexes were used for experiments.

Every effort was made to minimize animal suffering and to reduce the number of animals used. All animal experiments were performed in accordance with European Community Council Directive (2010/63/EU), and approved by the Ethics Committee for Animal Welfare (CEBA) of the University of the Basque Country.

\section{FIXATION AND TISSUE PROCESSING}

Rats were anesthetized with $6 \%$ chloral hydrate (performed under dim red light for DR groups), transcardially perfused with a fixative containing $4 \%$ paraformaldehyde in $0.1 \mathrm{M}$ phosphatebuffered saline (PBS), and the brains were stored overnight at $4^{\circ} \mathrm{C}$ in fresh fixative. The following day, a thick block of occipital cortex containing the visual area, and a thick block of medial cortex containing the primary somatosensory area, were coronally removed with a Rodent Brain Matrix (Electron Microscopic Sciences, Hatfield, UK) and rinsed in 0.1 M PBS for $4 \mathrm{~h}$. Samples for immunohistochemical analysis were embedded in paraffin, serially cut with a microtome into sections of $5 \mu \mathrm{m}$, and mounted on slides coated with 3-aminopropyltriethoxylane. On the other


FIGURE 1 | Different dark-rearing conditions used. (A) Dark-rearing with running wheel (exercise; DR-Ex); and (B) dark-rearing in an enriched environment and a running wheel (exercise; DR-EE-Ex). Litters were born in complete darkness and daily care tasks were performed under dim red light, as characterized in this figure. 
hand, samples assigned for volume analysis were stored in $30 \%$ sucrose in $0.1 \mathrm{M}$ PBS, until the tissues sank, were cut using a cryostat microtome in $50 \mu \mathrm{m}$ sections, and finally stored in free-floating chambers in $0.1 \mathrm{M}$ PBS.

\section{IMMUNOHISTOCHEMISTRY}

Paraffin sections were washed in 0.1 M PBS before inhibition of endogenous peroxidase activity using $4 \% \mathrm{H}_{2} \mathrm{O}_{2}$ in $0.1 \mathrm{M}$ PBS. Sections were washed in $0.1 \mathrm{M}$ PBS prior to the incubation with blocking solution [ $5 \%$ bovine serum albumin (BSA) in $0.1 \mathrm{M} \mathrm{PBS}$ ] for $1 \mathrm{~h}$. Then, an anti-S-100 $\beta$ polyclonal antibody (Ref. S2644; working dilution 1:200; Sigma-Aldrich, USA) was added overnight at $4^{\circ} \mathrm{C}$ in $5 \% \mathrm{BSA}$ in $0.1 \mathrm{M}$ PBS containing $0.3 \%$ Triton $\mathrm{X}-100$. Three washes in $0.1 \mathrm{M}$ PBS followed, and a biotinylated antirabbit secondary antibody (Elite ABC kit, Vector Laboratories, USA) was added for $2 \mathrm{~h}$ at room temperature. Once rinsed, the sections were incubated with an avidin-biotin-peroxidase complex (Elite ABC kit, Vector Laboratories). A reaction product was detected using diaminobenzidine $(0.25 \mathrm{mg} / \mathrm{ml})$ as the chromogen and hydrogen peroxide solution $(0.01 \%)$. Sections were processed with haematoxylin for the identification and localization of the primary visual cortex (V1) and the primary somatosensory cortex barrel field (S1BF). Sections were finally dehydrated and covered. As control for S-100 $\beta$, we used brains of rats and humans that had been subjected to trauma. Negative controls in which the primary antiserum was omitted were also included in each staining run.

\section{NISSL STAINING}

For Nissl staining, $50 \mu \mathrm{m}$-thick sections mounted on gelatinecoated slides were incubated in toluidine blue $\mathrm{pH} 4.1$ for $1 \mathrm{~min}$, dehydrated through a battery of alcohols with increased graduation, and coverslipped.

\section{QUANTITATIVE ANALYSIS}

To quantify any changes during development and to compare groups from both situations, a blind morphometric study was performed where the person who measured the sections did not know the particular characteristics of each case (neither the age of the rats nor whether they belonged to the experimental groups). To estimate the number of astrocytes per area, we counted the number of positive cell bodies present in an area delimited by a grid fixed in the eyepiece, excluding those intersected by the $X$ and $Y$ axes. The grid was a square with sides measuring $250 \mu \mathrm{m}$ and the total surface area was therefore $62,500 \mu \mathrm{m}^{2}$. The grid was randomly placed on the V1 between cortical layers III and $\mathrm{V}$, ensuring that layer IV was included. Measurements were also taken from a non-visual area, e.g., S1BF on layer IV. The areas were selected with the aid of Paxinos and Watson's (1998) Rat Brain Atlas. A total of 56 animals, of both sexes, aged between P21 and P63 were used for DR-Ex group ( $n=8$ per age). Data for another 56 animals corresponding to the DR-EE-Ex group were retrieved from our previous work (Argandoña et al., 2009). Astrocytes were recognized by their morphological characteristics, i.e., S-100 $\beta$-positive cell bodies with short positive cytoplasmic processes, and by their nuclear morphology. We did not count positive cells which did not fit the morphology of astrocytes.
Measurements of each slice of the cortex were carried out on both the right and left hemispheres (eight fields were assessed on each hemisphere in each of the 10 slices taken per animal, i.e., 160 fields per animal) and the mean value per animal was calculated.

In order to rule out the possibility that changes in astroglial density might be due to changes in volume, the whole volume of layer IV was calculated for each region (V1 and S1BF), age, and condition using the Cavalieri method (Howard and Reed, 2005; Schmitz and Hof, 2005) included in the stereological module incorporated into the Mercator Software package for Computer Assisted Stereology (Explora Nova, La Rochelle, France).

For this purpose, Nissl-stained sections were examined with a computer coupled to an Olympus BX41 microscope equipped with a motorized stage under the control of the Mercator software. Both V1 and S1BF, and the boundaries of layer IV, were delineated every 8th section using Paxinos and Watson's (1998) Rat Brain Atlas aided by the cytoarchitectonic characteristics of $\mathrm{V} 1$ and S1BF. The computer calculated the total volume by multiplying the area of the delineated ROIs by the serial section interval and the actual measured section thickness (Figure 2).

\section{STATISTICAL ANALYSIS}

All statistical analyses were performed using SPSS statistical software (version 19.0 for IBM, Spain). Prior to analysis, data were examined for normal distribution using the Kolmogorov-Smirnov test and for homogeneity of variances using Levene's test. The effects of the age period, experimental condition and their interaction were determined by two-way ANOVA with posthoc analysis (posthoc tests used the Bonferroni correction for equal variances or Tamhane's T2 correction for unequal variances). In order to explore the effects of experimental conditions in greater depth, differences between them within age periods were evaluated using the Student's $t$-test. Data are described as mean \pm SEM. Significance was declared at $p<0.05$.

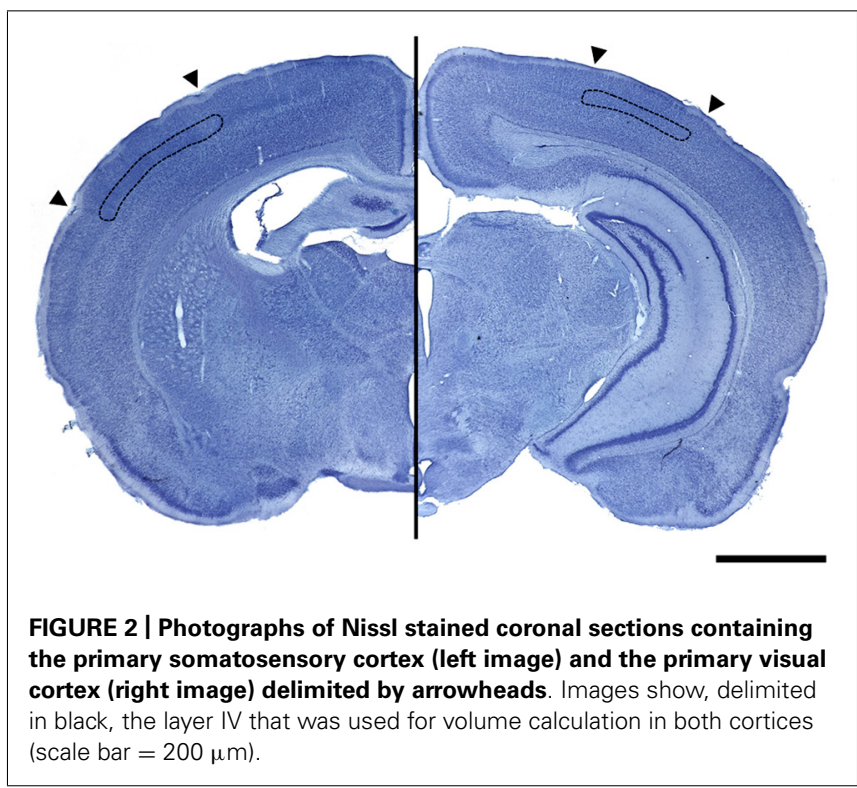





FIGURE 3 | S-100 $\beta$ staining shows that there are no morphological differences between astrocytes at any of the different ages and studied groups neither in the visual cortex nor in the somatosensory cortex. (A) P28 DR-Ex visual cortex; (B) P28 DR-EE-Ex visual cortex; (C) P56 DR-Ex visual cortex; (D) P56 DR-EE-Ex visual cortex; (E) P28 DR-Ex somatosensory cortex; (F) P28 DR-EE-Ex somatosensory cortex; (G) P56 DR-Ex somatosensory cortex; (H) P56 DR-EE-Ex somatosensory cortex (scale bar $=10 \mu \mathrm{m}$ ).

\section{RESULTS}

Immunoreactivity for S-100 $\beta$ was similar at all different ages both in the primary visual cortex and the primary somatosensory cortex, where strongly stained cell bodies and star-shaped processes were found across all cortical layers. No morphological differences between the studied groups were found at any of the different ages (Figure 3) and no quantitative difference could be guessed by microscopical analysis (Figure 4). Thus, we performed an unbiased stereological analysis in order to determine a numerical difference in astroglial population.

\section{ASTROGLIAL DENSITY}

The main result is a statistically significant difference in astroglial density of the DR-EE-Ex group compared to the DR-Ex group, in both visual and somatosensory cortices, and across all the studied ages.

\section{PRIMARY VISUAL CORTEX}

Two-way ANOVA revealed a significant interaction between age and experimental condition $(F=48.74$, $\mathrm{df}=6, p=0.000)$, as well as age $(F=26.88, \mathrm{df}=6, p=0.000)$ and condition effects $(F=1248.85, \mathrm{df}=1, p=0.000)$.

Student's $t$-test analysis was performed to study the differences between experimental conditions at each age period (Figure 5). Differences between DR-Ex and DR-EE-Ex groups were significant at all of ages analyzed $(p=0.000)$. The density of S-100 $\beta$ cells per area in the layer IV of the visual cortex was higher in the DR-EE-Ex group during the development with differences of $96 \%$ at P21 (11.70 \pm 0.35 and $22.95 \pm 0.59$, DR-Ex vs. DR-EE-Ex), $30 \%$ at P28 (15.18 \pm 0.37 and $19.60 \pm 0.43), 64 \%$ at P35 (12.03 \pm 0.33 and $19.72 \pm 0.47)$, $68 \%$ at $\mathrm{P} 42(11.64 \pm 0.28$ and $19.56 \pm 0.47), 90 \%$ at $\mathrm{P} 49$ $(10.79 \pm 0.33$ and $20.51 \pm 0.68), 54 \%$ at P56 (11.99 \pm 0.38 and $18.46 \pm 0.73)$ and $77 \%$ at P63 (11.06 \pm 0.33 and $19.55 \pm 0.49)$.

\section{PRIMARY SOMATOSENSORY CORTEX BARREL FIELD}

Two-way ANOVA revealed a significant interaction between age and experimental condition $(F=11.05$, $\mathrm{df}=6, p=0.000)$, as well as age $(F=28.06, \mathrm{df}=1, p=0.000)$ and condition effects $(F=485.71$, df $=1, p=0.000)$.

Student's $t$-test analysis between the two groups showed that, as in the visual cortex, differences were significant at all analyzed ages $(p=0.000)$, being DR-EE-Ex values higher than those for the DR-Ex group (Figure 5). Differences were 35\% at P21 $(15.44 \pm 0.41$ and $20.86 \pm 0.41$, DR-Ex vs. DR-EE-Ex), $52 \%$ at P28 $(12.87 \pm 0.32$ and $19.60 \pm 0.29), 18 \%$ at P35 (14.62 \pm 0.34 and $17.26 \pm 0.28), 31 \%$ at $\mathrm{P} 42$ (13.64 \pm 0.28 and $17.90 \pm 0.43), 23 \%$ at $\mathrm{P} 49$ (12.87 \pm 0.25 and $15.87 \pm 0.36), 24 \%$ at P56 (13.18 \pm 0.23 and $16.38 \pm 0.39)$ and $23 \%$ at P63 (13.49 \pm 0.25 and $16.56 \pm 0.41)$.

\section{CORTICAL VOLUME}

Dark-rearing affected the volume of visual cortex layer IV, which was significantly larger in animals reared in standard laboratory conditions with a 12-h light/dark cycle than in all dark-reared groups (Argandoña et al., 2009). As we described in a previous work, no difference was found between DR-Ex and DR-EE-Ex groups. On the contrary, in the somatosensory cortex, the volume of layer IV increased in dark-reared groups (Argandoña et al., 2009), although no significant differences were found between them. Cortical volume of both DR-Ex and DR-EE-Ex groups was also similar.

\section{PRIMARY VISUAL CORTEX VOLUME}

Two-way ANOVA revealed no differences or interactions between age and the experimental conditions $(F=1.59, \mathrm{df}=6, p=0.164)$, as well as any condition effects $(F=0.86, \mathrm{df}=1, p=0.357)$. However, age exerts significant changes over the primary visual cortex volume $(F=142.85, \mathrm{df}=6, p=0.000)$.

There were no significant differences between the DR-Ex and DR-EE-Ex groups' cortical volume at any of the studied 


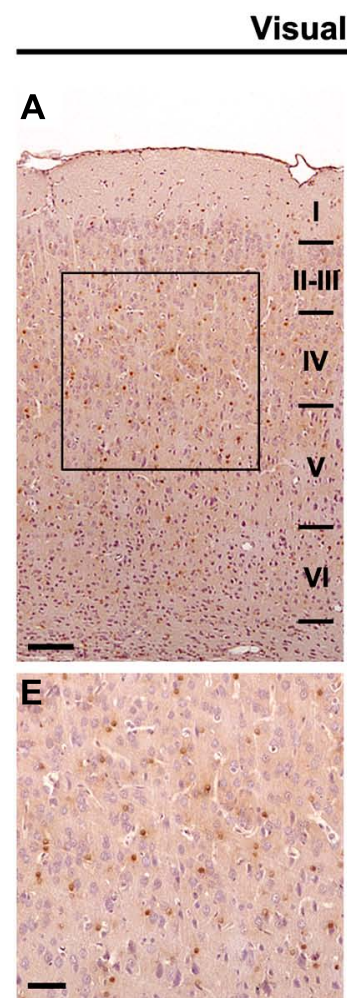

Visual cortex

B

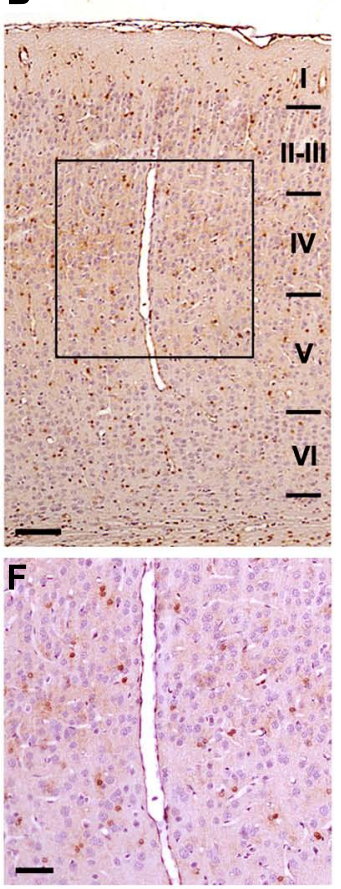

FIGURE 4 | Stereological analysis is required to discern astrocyte density differences between experimental conditions. Images at different magnifications express the need for stereological quantification in order to elucidate numerical differences. S-100 $\beta$ positive astrocytes:

\section{Somatosensory cortex}
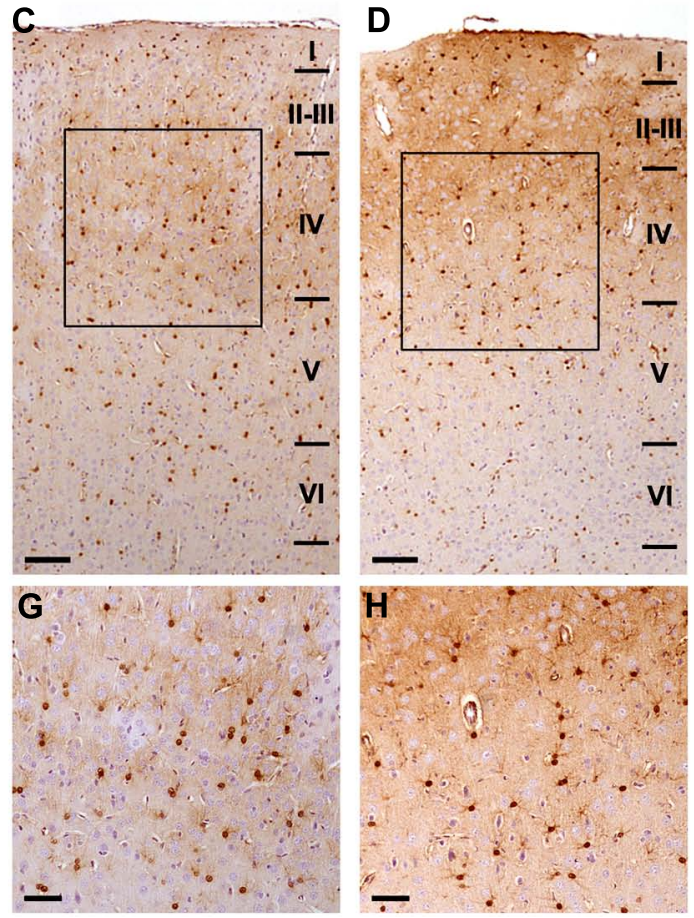

(A, E) P28 DR-Ex visual cortex; (B, F) P28 DR-EE-Ex visual cortex; (C, G) P28 DR-Ex somatosensory cortex; (D, H) P28 DR-EE-Ex somatosensory cortex (scale bar $=100 \mu \mathrm{m}$ in A, B, C, D; and $50 \mu \mathrm{m}$ in $\mathbf{E}$, $\mathbf{F}, \mathbf{G}, \mathbf{H})$. ages (Figure 5). Values were $0.576 \pm 0.049$ and $0.596 \pm 0.016$ (DR-Ex vs. DR-EE-Ex, $4 \%$ difference, $p=0.872$ ) at P21; $0.281 \pm 0.009$ and $0.297 \pm 0.007$ (6\% difference, $p=1.000)$ at P28; $0.277 \pm 0.010$ and $0.257 \pm 0.012(-7 \%$ difference, $p=0.649)$ at P35; $0.267 \pm 0.013$ and $0.257 \pm 0.010(-4 \%$ difference, $p=1.000)$ at $\mathrm{P} 42 ; 0.245 \pm 0.007$ and $0.247 \pm 0.011$ ( $1 \%$ difference, $p=1.000)$ at $\mathrm{P} 49 ; 0.265 \pm 0.009$ and $0.277 \pm 0.003$ (5\% difference, $p=1.000)$ at P56; and $0.260 \pm 0.011$ and $0.273 \pm 0.008$ (5\% difference, $p=0.904)$ at P63.

\section{PRIMARY SOMATOSENSORY CORTEX BARREL FIELD}

As occurred in the visual cortex, two-way ANOVA revealed no differences or interactions between age and the experimental conditions $(F=1.72, \mathrm{df}=6, p=0.129)$, as well as any condition effects $(F=2.59$, df $=1, p=0.112)$. However, age exerts significant changes over the primary visual cortex volume $(F=25.70$, $\mathrm{df}=6, p=0.000)$.

DR-Ex and DR-EE-Ex showed similar cortical values in the primary somatosensory cortex barrel field and statistically insignificant differences across all the ages studied (Figure 5). Values were $0.672 \pm 0.032$ and $0.632 \pm 0.027$ (DR-Ex vs. DR-EEEx, $-6 \%$ difference, $p=1.000)$ at $\mathrm{P} 21 ; 0.837 \pm 0.028$ and $0.884 \pm 0.050$ ( $6 \%$ difference, $p=0.648$ ) at $\mathrm{P} 28 ; 0.910 \pm 0.042$ and $0.905 \pm 0.055(-1 \%$ difference, $p=1.000)$ at $\mathrm{P} 35 ; 0.950 \pm 0.017$ and $0.973 \pm 0.033$ (2\% difference, $p=1.000)$ at $\mathrm{P} 42 ; 0.925 \pm 0.023$ and $0.948 \pm 0.050$ (3\% difference, $p=1.000)$ at $\mathrm{P} 49 ; 0.897 \pm 0.010$ and $0.936 \pm 0.038$ ( $4 \%$ difference, $p=0.075$ ) at P56; and $0.612 \pm 0.013$ and $0.2656 \pm 0.022$ ( $8 \%$ difference, $p=0.245$ ) at P63.

\section{DISCUSSION}

Visual deprivation induced by dark-rearing produces a downregulation of the astroglial population of the visual cortex, related to a delay in the development of the neurogliovascular unit elements (Bengoetxea et al., 2012). Our study shows that this effect can be partially reverted by a compensatory mechanism stimulating other areas such as the somatosensory and the motor cortices but, for a full recovery, a combination of both these strategies; i.e., environmental enrichment (somatosensorial implementation) and exercise (motor implementation), is required. Indeed, neither our previous results with cognitive enrichment (Argandoña et al., 2009; Ortuzar et al., 2013) nor our current results with physical enrichment are able to reach a reversion as deep as the one we attained with the combination (Argandoña et al., 2009). These results stand in accordance with a previous study on the hippocampus describing additive effects on adult neurogenesis of both components of the environmental enrichment (Fabel et al., 2009). A recent study also reported that, although physical exercise is the major neurogenic and neurotrophic stimulus most likely mediated by the angioglioneurin BDNF, cognitive 
A

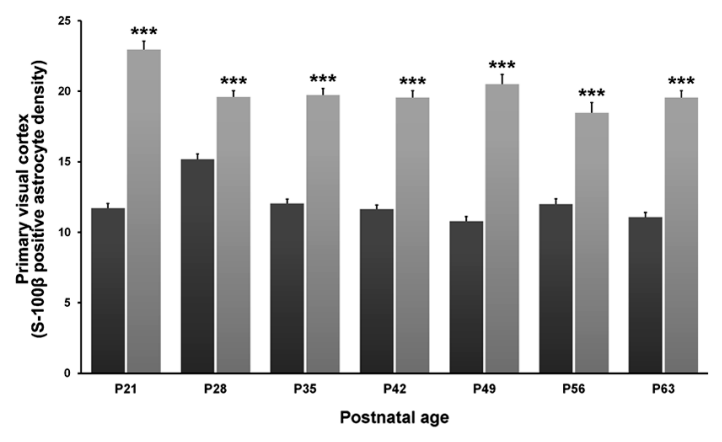

C

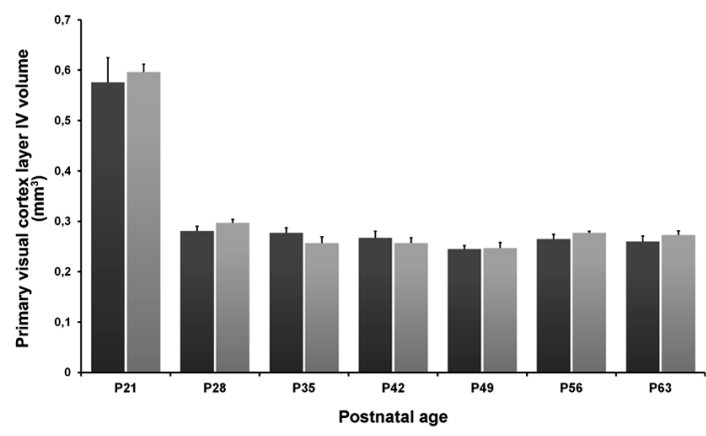

- DR-Ex

FIGURE 5 | Comparison of S-100 $\beta$ positive astrocyte density and cortical volume of the visual and somatosensory cortices between DR-Ex and DR-EE-Ex groups at each age considered. (A) Primary visual cortex layer IV S-100 $\beta$ positive astrocyte density and (B) primary somatosensory cortex barrel field S-100 $\beta$ positive astrocyte density. The horizontal axis shows the
B

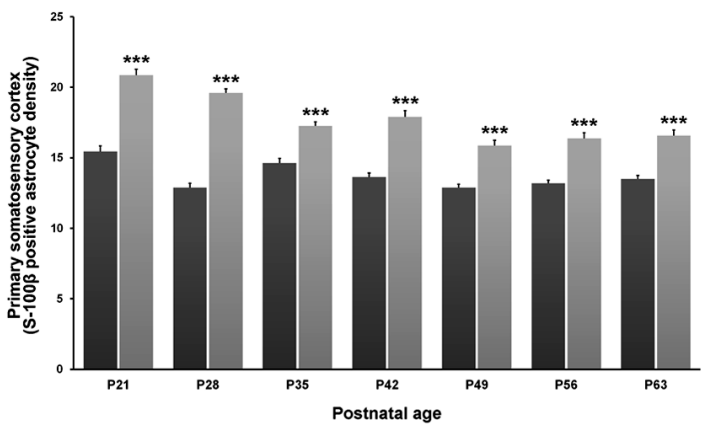

D

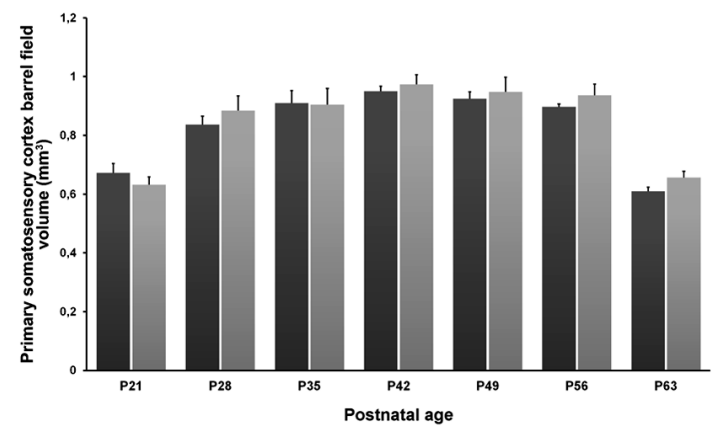

DR-EE-Ex

age of the animals. The vertical axis shows astrocyte density per $62500 \mu \mathrm{m}^{2}$ (mean \pm SEM; ${ }^{* * *} p \leq 0.001$ ). (C) Primary visual cortex layer IV volume $\left(\mathrm{mm}^{3}\right)$ and (D) primary somatosensory cortex barrel field volume $\left(\mathrm{mm}^{3}\right)$. The horizontal axis shows the age of the animals. The vertical axis shows the cortical volume in $\mathrm{mm}^{3}$ (mean \pm SEM).

At present, there is a broad consensus about the compulsory role of increased physical activity in enrichment models (Kobilo et al., 2011). Exercise activates molecular and cellular cascades that support and maintain brain plasticity, like BDNF, and promotes angiogenesis, neurogenesis, and functional changes in the neuronal structure (Berchtold et al., 2010). These effects are not restricted to the motor cortex as it might have been anticipated. They are also reflected in the entire cortical layer as well as in the hippocampus, improving, in this case, cognitive skills (Nithianantharajah and Hannan, 2009; Kerr et al., 2010; Lista and Sorrentino, 2010). Comparing data obtained from Argandoña et al. (2009) with our actual data, exercise counterbalances the deprivation effects produced by dark-rearing in the somatosensory cortex. Another point of interest is the difference between forced and voluntary exercise (Alomari et al., 2013). Previous studies have shown that forced exercise could be a source of stress which can mask the beneficial effects of exercise itself (Naylor et al., 2005; Griesbach et al., 2012). According to our observations, voluntary exercise is not limited to the use of the running wheel; there is also a high increase of physical activity when some elements such as platforms at different levels and tunnels connecting them are also included within the enriched environment. 

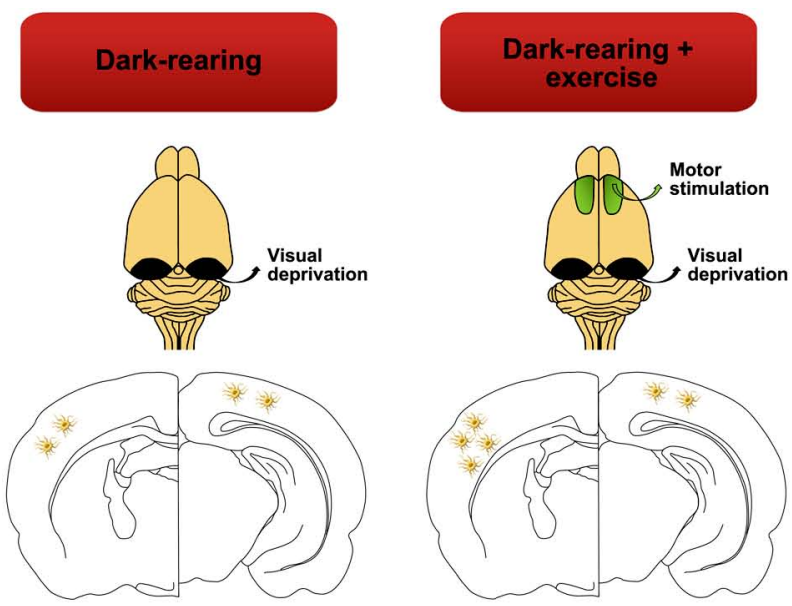

FIGURE 6 | Lack of visual stimulation (dark-rearing) not only exerts negative effects over the astrocyte density of the visual cortex but also affects other cortical areas such as the somatosensory cortex. Strategies such as exercise or environmental enrichment, by themselves, may vary and
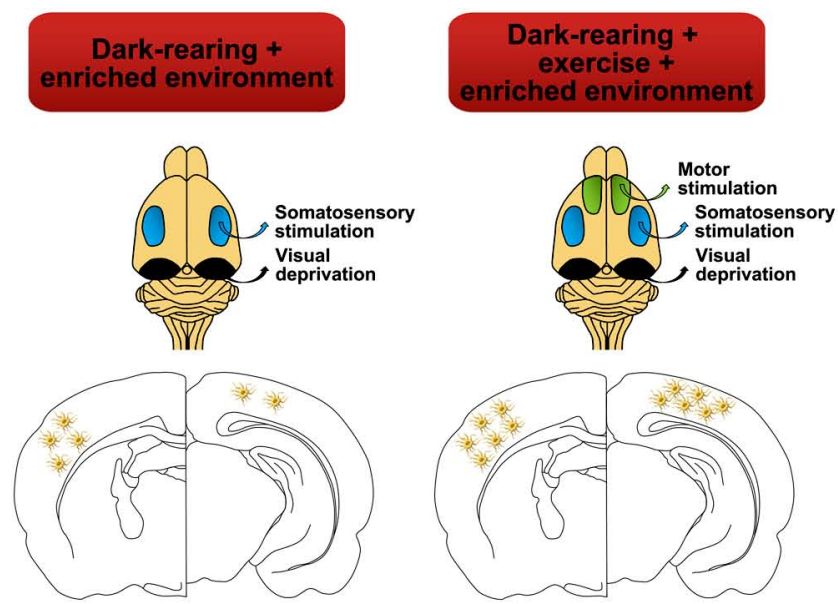

recover the density of astrocytes in the somatosensory cortex almost to control group levels (Argandoña et al., 2009). But it is the combination of both paradigms that significantly increases astroglial cell number and therefore reverses the negative effects of dark rearing in both cortices.
Whereas most of the environmental enrichment paradigms include the use of a running wheel as part of the enrichment, this could mask results for the purpose of studying specifically the visual stimulation. In this study, exercise has been dissociated to analyze the effect over the cortical astrocyte population of physical activity alone, or combined with an enriched environment. In previous studies we have demonstrated the visual exclusiveness of an environmental enrichment lacking exercise, which was unable to compensate for the decrease in vascular density (Bengoetxea et al., 2008) and astrocyte density (Argandoña et al., 2009) in the visual cortex. Interestingly, comparing our previous data to data from this work, both exercise and enrichment combined increase the astrocyte density of the somatosensory cortex in rats raised in darkness, reversing the effects of dark rearing and obtaining values similar to those of the control group.

In the absence of visual stimulus, the exploration of novelty objects with the aid of whiskers reflects on the somatosensory cortex and amplifies the effects over other sensory cortices such as the visual one. Our results are consistent with a report that demonstrated physical exercise alone was less effective than its combination with an enriched environment (Fabel et al., 2009) although some authors have indicated that the inclusion of a running wheel as a part of the enriched environment does not alter the effects produced throughout the brain (Pietropaolo et al., 2006). Thus, according to our results, a complete environmental enrichment paradigm should include shelters, wheels, toys, tunnels, platforms at different levels and a metallic network to allow for climbing.

The results of this work reinforce the data obtained previously (Argandoña et al., 2009). From the first stages of development, our results showed that the astroglial density in the DR-EE-Ex group, which combined both strategies, was statistically higher than in the control group. Neither exercise nor the enriched environment alone, reverted the negative effects induced by dark-rearing on the astroglial population, indicating that both strategies are complementary in producing cross-modal effects in the visual cortex (Figure 6). Moreover, we have observed that the increase in astroglial density is not attributable to an increase in volume. Dark-reared groups had a lower cortical volume than the control group, and the lack of difference between dark-reared groups means that any differences in astroglial density could not be attributable to variations in volume. Our results show a decrease in layer IV of the visual cortex volume in the control group at the beginning of the critical period which could be related to the decrease in the neuronal, vascular and astroglial densities (Argandoña and Lafuente, 2000; Argandoña et al., 2003, 2005; Heck et al., 2008). Although there is an increase in astroglial density in the DR-EE-Ex group, it does not induce any changes in volume. Our results agree with previous findings showing a similar volume of layer IV in the visual cortex of young adult control rats (Yates et al., 2008).

In our behavioral paradigm, we should take into account that forced exercise may represent an additional source of stress along with visual deprivation, which may interfere with our results. In rats, some authors have described relevant changes in astroglial population when animals are exposed to different stress situations. For example, Sharma et al. (1992) have described that heat stress causes hypertrophy and reactivation of astrocytes. On other hand, Araya-Callís et al. (2012), have also described that chronic psychosocial stress downregulates GFAP expression in the hippocampus. Nevertheless, we can discard any effect of stress on astroglial population. According to our previous experience on dark-rearing (Argandoña and Lafuente, 1996, 2000; Argandoña et al., 2003, 2005, 2009; Bengoetxea et al., 2008), as rats have been born in darkness and not introduced during development, no stress-induced behavioral changes have been detected.

Another point of interest is the observed difference in the somatosensory cortex. Visual deprivation encourages a greater use of the somatosensory skills, exerting effects on this cortex from P21 to P63. This increase is enhanced in environmentally enriched 
animals and the reinforcement is greater when the motor cortex is stimulated by the use of physical exercise. Unlike in the visual cortex, the volume of the somatosensory cortex of rats reared in darkness was greater than the control group ones, but no statistically significant difference was observed between them. The volume of the somatosensory cortex of dark-reared rats increased since the early stages of development due to the aforementioned compensation. As the critical period for the somatosensory cortex occurs earlier than the visual one (during the first week of life), the effects of compensatory skills through the use of whiskers are noticeable right from the beginning of the study. In a recent paper, in human blind subjects, Weaver et al. (2013) have suggested that a high level of cross-modal activity not merely prevents the decrease in astrocyte levels that is normally seen in dark-reared animals, but might even have an effect analogous to the effects of environmental enrichment, resulting in an increase in the astrocyte population local to the occipital cortex. They also have described neurochemical differences between blind and sighted subjects such as higher levels of GABA, lower levels of choline and higher levels of creatine. All these results support the idea that neurovascular coupling is also altered by early blindness. Astrocytes are not only a major component of the neurogliovascular unit, inducing and maintaining $\mathrm{BBB}$ properties and releasing angioglioneurins (Argandoña et al., 2012), they also play a major role in synaptic transmission. In the last 25 years, many studies have revealed the existence of bidirectional communication between neurons and astrocytes and proposed the term "tripartite synapse" to indicate

\section{REFERENCES}

Alomari, M. A., Khabour, O. F., Alzoubi, K. H., and Alzubi, M. A. (2013). Forced and voluntary exercises equally improve spatial learning and memory and hippocampal BDNF levels. Behav. Brain Res. 247, 34-39. doi: 10.1016/j.bbr.2013.03.007

Araque, A., Parpura, V., Sanzgiri, R. P., and Haydon, P. G. (1999). Tripartite synapses: glia, the unacknowledged partner. Trends Neurosci. 22, 208-215. doi: 10.1016/S01662236(98)01349-6

Araya-Callís, C., Hiemke, C., Abumaria, N., and Flugge, G. (2012). Chronic psychosocial stress and citalopram modulate the expression of the glial proteins GFAP and NDRG2 in the hippocampus. Psychopharmacology 224, 209-222. doi: 10.1007/s00213-0122741-x

Argandoña, E. G., Bengoetxea, H., and Lafuente, J. V. (2005). Lack of experience-mediated differences in the immunohistochemical expression of blood-brain barrier markers (EBA and GluT-1) during the postnatal development of the rat visual cortex. Brain Res. Dev. Brain Res. 156, 158-166. doi: 10.1016/j.devbrainres.2005.02.007
Argandoña, E. G., Bengoetxea, H., and Lafuente, J. V. (2009). Physical exercise is required for environmental enrichment to offset the quantitative effects of dark-rearing on the S-100beta astrocytic density in the rat visual cortex. J. Anat 215, 132-140. doi: 10.1111/j.14697580.2009.01103.x

Argandoña, E. G., Bengoetxea, H., Ortuzar, N., Bulnes, S., RicoBarrio, I., and Lafuente, J. V. (2012). Vascular endothelial growth factor: adaptive changes in the neuroglialvascular unit. Curr. Neurovasc. Res. 9, 72-81. doi: 10.2174/15672021 2799297119

Argandoña, E. G., and Lafuente, J. V. (1996). Effects of dark-rearing on the vascularization of the developmental rat visual cortex. Brain Res. 732, 43-51. doi: 10.1016/00068993(96)00485-4

Argandoña, E. G., and Lafuente, J. V. (2000). Influence of visual experience deprivation on the postnatal development of the microvascular bed in layer IV of the rat visual cortex. Brain Res. 855 137-142. doi: 10.1016/S0006-8993 (99)02361-6

Argandoña, E. G., Rossi, M. L., and Lafuente, J. V. (2003). Visual deprivation effects on the slo0beta

the astrocyte as the third element of the synapse (Araque et al., 1999), therefore, any element that interfere with cortical function, such as visual deprivation, modifies all the elements of the neurogliovascular unit, also inducing changes in the tripartite synapse.

\section{CONCLUSION}

Exposure to deprived inputs has serious consequences during the postnatal development of the sensory cortices, which can be reverted by reactivating cortical plasticity using physiological strategies such as enriched environment and voluntary physical exercise. There is a cross-modal plasticity that leads to a compensatory upregulation of the nondeprived senses that even rescues the cortical deficits of the deprived one. Our main result shows that somatosensorial and physical stimuli have effects all over the cortex and can rescue the deficits of the astroglial population of the visual cortex produced by the dark-rearing. Neither of these two strategies is enough by itself to offset the decrease on the astroglial population. However, this can be reverted by a combination of the two.

\section{ACKNOWLEDGMENTS}

This study has been supported by SAIOTEK (Industry Dept., Government of the Basque Country), IT/794/13 (Basque Government), UFI 11/32 (UPV/EHU) and Jesus Gangoiti Barrera Foundation. Naiara Ortuzar is supported by a postdoctoral grant of the University of the Basque Country (UPV/EHU).

positive astrocytic population in the developing rat visual cortex: a quantitative study. Brain Res. Dev. Brain Res. 141, 6369. doi: 10.1016/S0165-3806(02) 00643-0

Bartoletti, A., Medini, P., Berardi, N. and Maffei, L. (2004). Environmental enrichment prevents effects of dark-rearing in the rat visual cortex. Nat. Neurosci. 7, 215-216. doi: 10.1038/nn1201

Bechara, R. G., and Kelly, Á. M. (2013). Exercise improves object recognition memory and induces BDNF expression and cell proliferation in cognitively enriched rats. Behav. Brain Res. 245, 96-100. doi: 10.1016/j.bbr.2013. 02.018

Ben Achour, S., and Pascual, O. (2012). Astrocyte-neuron communication: functional consequences. Neurochem. Res. 37, 2464-2473. doi: 10.1007/s11064-012-0807-0

Bengoetxea, H., Argandoña, E. G. and Lafuente, J. V. (2008). Effects of visual experience on vascular endothelial growth factor expression during the postnatal development of the rat visual cortex. Cereb. Cortex 18, 1630-1639. doi: 10.1093/cercor/ bhm 190

Bengoetxea, H., Ortuzar, N., Bulnes, S., Rico-Barrio, I., Lafuente, J. V., and
Argandoña, E. G. (2012). Enriched and deprived sensory experience induces structural changes and rewires connectivity during the postnatal development of the brain. Neural Plast. 2012, 305693. doi: 10.1155/2012/305693

Berchtold, N. C., Castello, N., and Cotman, C. W. (2010). Exercise and time-dependent benefits to learning and memory. Neuroscience 167, 588-597. doi: 10.1016/j.neuroscience.2010.02.050

Burrows, E. L., McOmish, C. E., and Hannan, A. J. (2011). Geneenvironment interactions and construct validity in preclinical models of psychiatric disorders. Prog. Neuropsychopharmacol. Biol. Psychiatry 35, 1376-1382. doi: 10.1016/j.pnpbp.2010.12.011

During, M. J., and Cao, L. (2006). VEGF, a mediator of the effect of experience on hippocampal neurogenesis. Curr. Alzheimer Res. 3, 29-33. doi: 10.2174/156720506775697133

Fabel, K., Wolf, S. A., Ehninger, D., Babu, H., Leal-Galicia, P., and Kempermann, G. (2009). Additive effects of physical exercise and environmental enrichment on adult hippocampal neurogenesis in mice. Front. Neurosci. 3:50. doi: 10.3389/neuro.22.002.2009 
Fagiolini, M., Pizzorusso, T., Berardi, N., Domenici, L., and Maffei, L. (1994). Functional postnatal development of the rat primary visual cortex and the role of visual experience: dark rearing and monocular deprivation. Vis. Res. 34, 709720. doi: $10.1016 / 0042-6989(94)$ 90210-0

Figley, C. R., and Stroman, P. W. (2011). The role(s) of astrocytes and astrocyte activity in neurometabolism, neurovascular coupling, and the production of functional neuroimaging signals. Eur. J. Neurosci. 33, 577-588. doi: 10.1111/j.14609568.2010.07584.x

Frasnelli, J., Collignon, O., Voss, P., and Lepore, F. (2011). Crossmodal plasticity in sensory loss. Prog. Brain Res. 191, 233-249. doi: 10.1016/B978-0444-53752-2.00002-3

Greenough, W. T., Black, J. E., and Wallace, C. S. (1987). Experience and brain development. Child Dev. 58, 539-559. doi: 10.2307/1130197

Griesbach, G. S., Tio, D. L., Vincelli, J., McArthur, D. L., and Taylor, A. N. (2012). Differential effects of voluntary and forced exercise on stress responses after traumatic brain injury. J. Neurotrauma 29, 1426-1433. doi: 10.1089/neu. 2011.2229

Heck, N., Golbs, A., Riedemann, T., Sun, J. J., Lessmann, V., and Luhmann, H. J. (2008). Activity-dependent regulation of neuronal apoptosis in neonatal mouse cerebral cortex. Cereb. Cortex 18, 1335-1349. doi: 10.1093/ cercor/bhm165

Hensch, T. K. (2004). Critical period regulation. Annu. Rev. Neurosci. 27, 549-579. doi: 10.1146/annurev. neuro.27.070203.144327

Hensch, T. K. (2005). Critical period plasticity in local cortical circuits. Nat. Rev. Neurosci. 6, 877-888. doi: 10.1038/nrn1787

Hensch, T. K., and Fagiolini, M. (2005). Excitatory-inhibitory balance and critical period plasticity in developing visual cortex. Prog. Brain Res. 147, 115-124. doi: 10.1016/S00796123(04)47009-5

Howard, C. V., and Reed, M. G. (2005). Unbiased Stereology: Three-Dimensional Measurement in Microscopy, 2nd Edn. Oxford: Garland Science/Bios Scientific Publishers.

Kerr, A. L., Steuer, E. L., Pochtarev, V., and Swain, R. A. (2010). Angiogenesis but not neurogenesis is critical for normal learning and memory acquisition. Neuroscience 171, 214-226. doi: 10.1016/j.neuroscience.2010.08.008
Kobilo, T., Liu, Q. R., Gandhi, K., Mughal, M., Shaham, Y., and van Praag, H. (2011). Running is the neurogenic and neurotrophic stimulus in environmental enrichment. Learn. Mem. 18, 605-609. doi: 10.1101/lm.2283011

Krech, D., Rosenzweig, M. R., and Bennett, E. L. (1960). Effects of environmental complexity, and training on brain chemistry. J. Comp. Physiol. Psychol. 53, 509-519. doi: 10.1037/h0045402

Lafuente, J. V., Ortuzar, N., Bengoetxea, H., Bulnes, S., and Argandoña, E. G. (2012). Vascular endothelial growth factor and other angioglioneurins: key molecules in brain development and restoration. Int. Rev. Neurobiol. 102, 317-346. doi: 10.1016/B978-012-386986-9.00012-0

Leggio, M. G., Mandolesi, L., Federico, F., Spirito, F., Ricci, B., Gelfo, F., et al. (2005). Environmental enrichment promotes improved spatial abilities and enhanced dendritic growth in the rat. Behav. Brain Res. 163, 78-90. doi: 10.1016/j.bbr.2005.04.009

Levelt, C. N., and Hübener, M. (2012) Critical-period plasticity in the visual cortex. Annu. Rev. Neurosci. 35, 309330. doi: 10.1146/annurev-neuro061010-113813

Lista, I., and Sorrentino, G. (2010). Biological mechanisms of physical activity in preventing cognitive decline. Cell Mol. Neurobiol. 30, 493-503. doi 10.1007/s10571-009-9488-x

McGann, J. C., Lioy, D. T., and Mandel, G. (2012). Astrocytes conspire with neurons during progression of neurological disease. Curr Opin. Neurobiol. 22, 850-858. doi: 10.1016/j.conb.2012.03.009

Merabet, L. B., and Pascual-Leone, A. (2010). Neural reorganization following sensory loss: the opportunity of change. Nat. Rev. Neurosci. 11, 44-52. doi: 10.1038/nrn2758

Mustroph, M. L., Chen, S., Desai, S. C., Cay, E. B., DeYoung, E K., and Rhodes, J. S. (2012). Aerobic exercise is the critical variable in an enriched environment that increases hippocampal neurogenesis and water maze learning in male C57BL/6J mice. Neuroscience 219, 62-71. doi: 10.1016/j.neuroscience. 2012.06.007

Naylor, A. S., Persson, A. I., Eriksson, P. S., Jonsdottir, I. H., and Thorlin, T. (2005). Extended voluntary running inhibits exercise-induced adult hippocampal progenitor proliferation in the spontaneously hypertensive rat. J. Neurophysiol. 93, 2406-2414. doi: 10.1152/ jn. 01085.2004
Nithianantharajah, J., and Hannan, A. J. (2009). The neurobiology of brain and cognitive reserve: mental and physical activity as modulators of brain disorders. Prog. Neurobiol. 89, 369-382. doi: 10.1016/j.pneurobio.2009.10.001

Ortuzar, N., Argandoña, E. G., Bengoetxea, H., and Lafuente, J. V. (2011). Combination of intracortically administered VEGF and environmental enrichment enhances brain protection in developing rats. J. Neural Transm. 118, 135-144. doi: 10.1007/s00702-010-0496-2

Ortuzar, N., Argandoña, E. G., Bengoetxea, H., Leis, O., Bulnes, S. and Lafuente, J. V. (2010). Effects of VEGF administration or neutralization on the BBB of developing rat brain. Acta Neurochir. Suppl. 106, 55-59. doi: 10.1007/978-3-21198811-4_9

Ortuzar, N., Rico-Barrio, I., Bengoetxea, H., Argandoña, E. G., and Lafuente, J. V. (2013). VEGF reverts the cognitive impairment induced by a focal traumatic brain injury during the development of rats raised under environmental enrichment. Behav. Brain Res. 246, 36-46. doi: 10.1016/j. bbr.2013.02.036

Pang, T. Y., and Hannan, A. J. (2013). Enhancement of cognitive function in models of brain disease through environmental enrichment and physical activity. Neuropharmacology 64, 515-528. doi: 10.1016/j.neuropharm.2012.06.029

Paxinos, G., and Watson, C. (1998). The Rat Brain in Stereotaxic Coordinates. San Diego: Academic Press.

Perea, G., Navarrete, M., and Araque, A. (2009). Tripartite synapses: astrocytes process and control synaptic information. Trends Neurosci. 32, 421-431. doi: 10.1016/j.tins. 2009.05.001

Peterson, E. C., Wang, Z., and Britz, G. (2011). Regulation of cerebral blood flow. Int. J. Vasc. Med. 2011, 823525. doi: 10.1155/2011/823525

Pietropaolo, S., Feldon, J., Alleva, E., Cirulli, F., and Yee, B. K. (2006). The role of voluntary exercise in enriched rearing: a behavioral analysis. Behav. Neurosci. 120, 787-803. doi: 10.1037/0735-7044.120.4.787

Rauschecker, J. P. (1995). Compensatory plasticity and sensory substitution in the cerebral cortex. Trends Neurosci. 18, 36-43. doi: 10.1016/0166-2236(95)93948-W

Schmitz, C., and Hof, P. R. (2005). Design-based stereology in neuroscience. Neuroscience 130, 813831. doi: 10.1016/j.neuroscience. 2004.08.050
Sharma, H. S., Zimmer, C., Westman, J., and Cervós-Navarro, J. (1992). Acute systemic heat stress increases glial fibrillary acidic protein immunoreactivity in brain: experimental observations in conscious normotensive young rats. Neuroscience 48, 889901. doi: 10.1016/0306-4522(92) 90277-9

Simpson, J., and Kelly, J. P. (2011). The impact of environmental enrichment in laboratory rats - behavioural and neurochemical aspects. Behav. Brain Res. 222, 246-264. doi: 10.1016/j.bbr.2011.04.002

Sirevaag, A. M., and Greenough, W. T. (1991). Plasticity of GFAPimmunoreactive astrocyte size and number in visual cortex of rats reared in complex environments. Brain Res. 540, 273-278. doi: 10.1016/00068993(91)90517-Y

Weaver, K. E., Richards, T. L., Saenz, M., Petropoulos, H., and Fine, I. (2013). Neurochemical changes within human early blind occipital cortex. Neuroscience doi: 10.1016/j.neuroscience.2013.08.004 [Epub ahead of print].

Will, B., Galani, R., Kelche, C., and Rosenzweig, M. R. (2004). Recovery from brain injury in animals: relative efficacy of environmental enrichment, physical exercise or formal training (1990-2002). Prog. Neurobiol. 72, 167-182. doi: 10.1016/j.pneurobio. 2004.03.001

Xie, H., Wu, Y., Jia, J., Liu, G., Zhang, Q., Yu, K., et al. (2013). Enrichmentinduced exercise to quantify the effect of different housing conditions: a tool to standardize enriched environment protocols. Behav. Brain Res. 249, 81-89. doi: 10.1016/j.bbr.2013. 04.032

Yates, M. A., Markham, J. A., Anderson, S. E., Morris, J. R., and Juraska, J. M. (2008). Regional variability in age-related loss of neurons from the primary visual cortex and medial prefrontal cortex of male and female rats. Brain Res. 1218, 1-12. doi: 10.1016/j.brainres. 2008.04.055

Zacchigna, S., Lambrechts, D., and Carmeliet, P. (2008). Neurovascular signalling defects in neurodegeneration. Nat. Rev. Neurosci. 9, 169-181. doi: 10.1038/nrn 2336

Conflict of Interest Statement: The authors declare that the research was conducted in the absence of any commercial or financial relationships that could be construed as a potential conflict of interest. 
Received: 15 May 2013; accepted: 11 September 2013; published online: 04 October 2013.

Citation: Bengoetxea H, Ortuzar N, Rico-Barrio I, Vicente Lafuente J and Argandoña EG (2013) Increased physical activity is not enough to recover astrocytic population from dark-rearing. Synergy with multisensory enrichment is required. Front. Cell. Neurosci. 7:170 doi: 10.3389/fncel.2013.00170

This article was submitted to the journal Frontiers in Cellular Neuroscience.
Copyright (C) 2013 Bengoetxea, Ortuzar, Rico-Barrio, Vicente Lafuente and Argandoña. This is an open-access article distributed under the terms of the Creative Commons Attribution License (CC BY). The use, distribution or reproduction in other forums is permitted provided the original author(s) or licensor are credited and that the original publication in this journal is cited, in accordance with accepted academic practice. No use, distribution or reproduction is permitted which does not comply with these terms. 